\title{
DYNAMICAL FRICTION IN CLUSTERS OF GALAXIES
}

Simon D.M. White

Institute of Astronomy, Madingley Road, Cambridge, England

La formule classique pour la friction dynamique prédit que l'évolution de l'orbite d'une galaxie qui est membre d'un amas sera trop rapide pour être en concordance avec les observations, si toute la masse de l'amas est concentrée dans les galaxies elles-mêmes. Quoique cette formule ait été prouvée sous des conditions très différentes, elle semble bien décrire les effets des rencontres gravitationelles dans un amas qui est stabilisé par sa propre gravitation. La friction dynamique mène à une concentration de galaxies massives au centre d'un amas, laquelle concentration doit avoir des effets importants sur la structure de la galaxie centrale. On ignore tout des détails de l'évolution dynamique de cette galaxie, mais $i 1$ est probable que les effets soient assez grands pour interdire toute déduction cosmologique de la relation $\mathrm{m}-\mathrm{Z}$.

The concept of dynamical friction was introduced into stellar dynamics by Chandrasekhar (1943) who used it to study the evolution of star clusters. He showed that the dominant effect of gravitational encounters on a massive particle moving with velocity $\underline{v}$ through a uniform isotropic gas of lighter particles is that of a resisting force

$$
\underline{F}=-4 \pi G^{2} m^{2} \frac{\underline{v}}{|\underline{v}|^{3}} \ln \Lambda \rho(|\underline{v}|)
$$

where $m$ is the mass of the heavy particle, $\rho(|\underline{v}|)$ is the mass density of background objects with speeds less than $|\underline{v}|$, and $\Lambda$ is the ratio of the maximum and minimum impact parameters for which encounters are effective. Although Chandrasekhar derived equation (1) for rectilinear motion through a uniform medium, it has usually been applied to periodic orbits in a non-uniform self-gravitating system. Thus it has recently 
been used to describe the evolution of the globular cluster population in the halo of a galaxy (Tremaine, Ostriker \& Spitzer 1975, Tremaine 1976b), of the orbits of satellites around giant galaxies (Tremaine 1976a) and of the distribution of galaxies in a rich cluster (Lecar 1974, Ostriker and Tremaine 1975, White 1976a). In such situations equation ( 1 ) predicts that collisional relaxation will cause the orbits of heavy objects to shrink at rates proportional to their masses, and hence that material will pile up at the centre of the system. This central excess is identified with the nucleus in an individual galaxy or with the extended central galaxy in a rich cluster.

The evolutionary time-scales implied by equation (1) can be estimated from a simple model. Let us assume that the background sea of light particles contributes the bulk of the cluster mass and can be roughly model led by a singular isothermal sphere with one-dimensional velocity dispersion $\sigma$ and density $\rho(r)=\sigma^{2} / 2 \pi G r^{2}$. We can define a frictional relaxation time for a heavy particle in an orbit of mean radius $\bar{r}$ by $T_{f}=\bar{r} /(d \bar{r} / d t)$. Then for an orbit of the average eccentricity equation (1) leads to

$$
T_{0} / T_{f} \simeq 4 \ln \Lambda(m / M(\bar{r}))
$$

where $T_{0}$ is the orbital period, $m$ is the mass of the particle and $M(\bar{r})$ is the cluster mass within radius $\bar{r}$. Appropriate values for in $\Lambda$ lie in the range 3 - 6 for the problems described above, so that relaxation effects will occur on the order of the orbital time if $\mathrm{m} / \mathrm{M}$ is greater than a few per cent. The brightest galaxies in a cluster usually contain one or two per cent of the total luminosity, and so we would expect to see most of them sitting in the cluster centre if their mass-tolight ratio were the same as that of the cluster as a whole. This is quite inconsistent with observation.

It is not clear that the application of equation (1) to periodic 
orbits in a self-gravitating system is justified - Jupiter, for example, does not lose energy to the other planets in the Solar System at the rate suggested by a naive application of the theory. Attempts to derive a formula like equation (1) by considering strictly periodic orbits usually conclude that when resonances can be ignored the response of the background reaches a steady state in which there is no nett frictional force on the orbiting particle (e.g. Kalnajs 1970). It is possible to check the validity of the dynamical friction formula in a selfgravitating system by comparing its predictions with direct integrations of the $\mathrm{N}$-body equations of motion. A recent 700-body simulation of the formation and evolution of a galaxy cluster showed a degree of masssegregation which agreed well with that predicted by equation (1) (White 1976b, 1976c). Similarly other calculations have shown that the relaxation time-scale in $\mathrm{N}$-body models of star clusters is close to the classical relaxation time derived by Chandrasekhar (1942) in a similar way to equation (1). (See the review by Aarseth and Lecar 1975). The dynamical friction formula is thus vindicated in at least one limit. Detailed comparison of the strong segregation which developed in the above 700-body model with the weak segregation observed in real clusters confirms the conclusion of the previous paragraph that the mass-to-light ratio of the bright galaxies in a rich cluster must be much less than that of the cluster as a whole (White 1976c); such clusters must be bound by intergalactic material. Nevertheless we expect that equation (1) will still describe the interaction of the galaxies with the intergalactic medium, since the implied rates of evolution are almost certainly too large for the collective response of the system to have time to reach the symmetric steady state in which friction disappears.

The lack of strong segregation in observed clusters implies that the distribution of faint galaxies can have been little affected by dynamical friction. This distribution is therefore determined by the process of cluster formation, and hence it is plausible that the material which binds the cluster is similarly distributed. White (1976a) calculated detailed models for the evolution of the galaxy distribution 


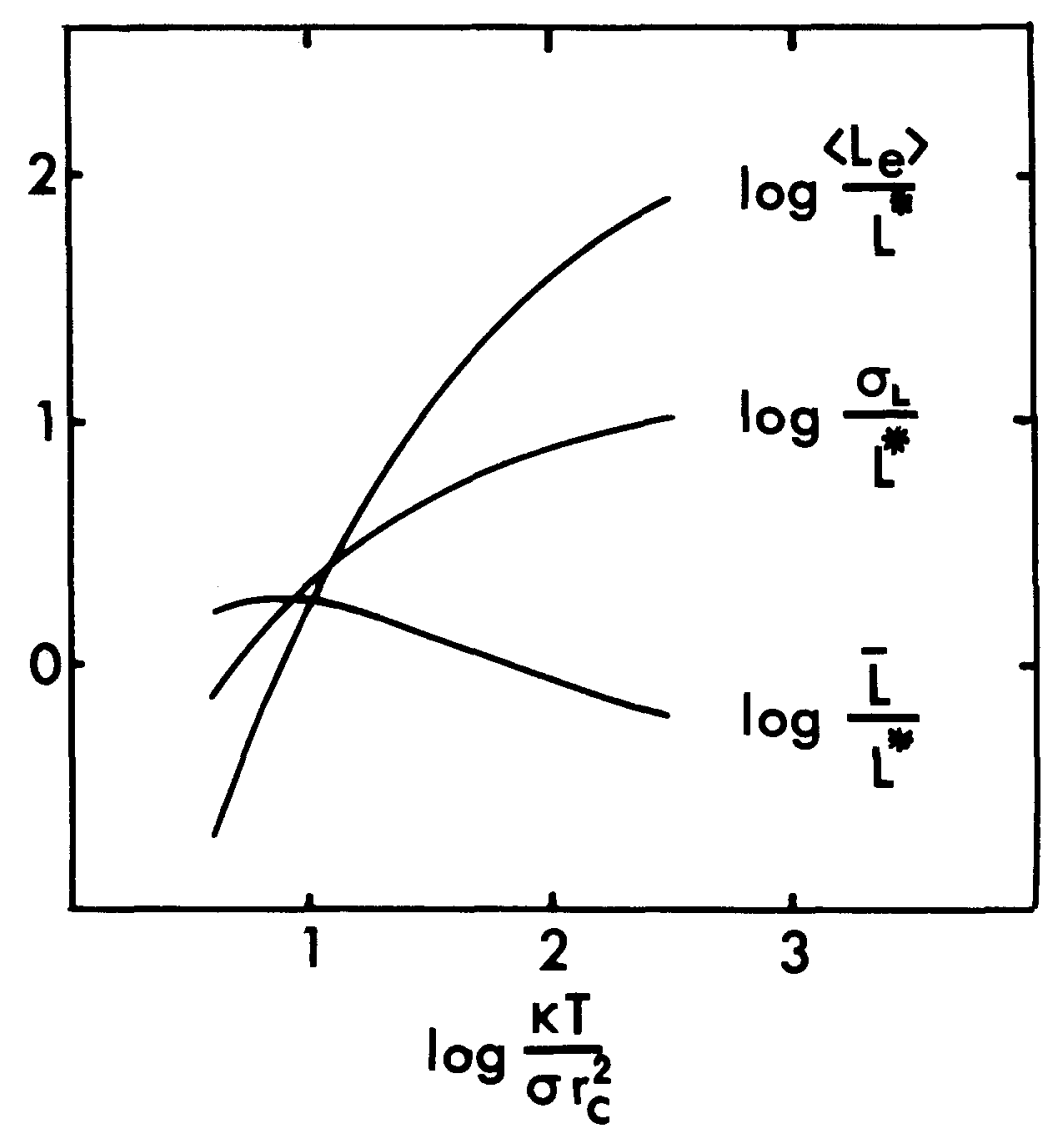

Fig. 1. The properties of the central accumulation of luminosity in the isothermal model of a galaxy cluster described in the text. In this model the line of sight velocity dispersion is $1000 \sigma \mathrm{km} / \mathrm{sec}$, the core radius is $240 r_{c} k p c$ and the initial projected core luminosity, $L_{c}$, is $25 \mathrm{~L}^{*}$. The effective time of evolution is $10^{10} \mathrm{~T}$ years and the galactic mass-to-light ratio, $k$ is in solar units. The expected luminosity of the central accumulation is $\left\langle L_{e}\right\rangle$, $\sigma_{L}$ is the dispersion in this luminosity due to the randomness of the initial galaxy distribution and $\bar{L}$ is the mean luminosity of the galaxies from which it is built up. For different values of $L_{c},\left\langle L_{e}\right\rangle$ scales as $L_{c}, \sigma_{L}$ as $L_{C}^{\frac{1}{2}}$ and $\bar{L}$ is unchanged.

in rich clusters based on the assumption that both the galaxies and the "missing mass" were initially distributed in an isothermal sphere with line of sight velocity dispersion $\sigma$ and core radius $r_{c}$. The masses of the galaxies were taken to be $\kappa$ times their luminosities and following Schechter (1976) a luminosity function of the form

$$
n(L) \frac{d L}{L^{\star}}=N^{*}\left(L / L^{*}\right)^{-5 / 4} \exp \left(-L / L^{*}\right) d L / L^{*}
$$


was adopted with $L_{v}^{*}=4 \times 10^{10} L_{\odot}$. (We take $H_{0}=50 \mathrm{~km} / \mathrm{sec} / \mathrm{Mpc}$ here and below). As this model evolves, bright galaxies drift inwards and pile up at the centre. The properties of the predicted central accumulation are shown in Fig. 1 as a function of the cluster parameters and of the effective time of evolution. It is clear that to avoid an altogether unpalatable amount of material from reaching the cluster centre in the available time, $\kappa$ must be quite small. Data on the Coma cluster appear to require $k<30$ (White 1976c). The mean luminosity $\bar{L}$ of the galaxies which reach the centre is greater than $L^{*}$ for acceptable values of the total central excess, $\left\langle\mathrm{L}_{\mathrm{e}}\right\rangle$; the central galaxy in a rich cluster is therefore more likely to increase its luminosity by absorbing another of the bright galaxies in the cluster than by absorbing several fainter objects. For this reason the purely statistical dispersion, $\sigma_{L}$, predicted for the accreted luminosity is quite large. In addition, since $\left\langle L_{e}>/ L_{c}\right.$ is a strong function of $k T / \sigma r_{c}{ }^{2}$, a substantial contribution to any observed dispersion in accreted luminosity is expected from differences in the parameters appropriate to different clusters.

At first sight it is difficult to reconcile the extent of evolution predicted by Fig. I with present observations which show both a small dispersion and a weak dependence on cluster richness of the magnitudes of brightest cluster members (Sandage 1973, 1975; Gunn and Oke 1975). The weak richness dependence is not necessarily in contradiction with the above analysis which does not predict any simple dependence of accreted luminosity on cluster richness. The small dispersion in magnitudes is less easy to explain, but it must be stressed that the central excess of luminosity predicted by Fig. 1 cannot be identified with the light appearing within a certain fixed distance of the brightest galaxy in a cluster. Dynamical friction arguments merely indicate the expected number and luminosity distribution of galaxies which will sink into the central regions; they cannot describe what happens when the galaxies get there. As was originally noted by Ostriker and Tremaine (1975) and was more extensively demonstrated by Gunn and Tinsley (1976), 
the structure of the object which results from the merging of two galaxies depends strongly on the initial structure of the galaxies involved and on the details of their amalgamation. The small dispersion in the observed magnitudes of brightest cluster members shows that the dependence of the luminosity within a certain metric diameter upon the amount of accreted material is not simple, and that it is weaker than might naively be expected. The total luminosities of the central galaxies in clusters are often substantially larger than their standard metric luminosities because such galaxies tend to possess extended envelopes. To a limiting visual isophote of $25 \mathrm{mag} / \mathrm{sq}$.arc sec. the two giant galaxies in the core of the Coma cluster have luminosities of $8 L^{*}$ and $5 L^{*}$ (Godwin and Peach 1976) and the CD galaxy in Abel1 1413 has a luminosity of $18 L^{*}$ (Austin and Peach 1974). Oemler (1973) found a very large halo surrounding the central galaxy in Abe 112670 which gives it a total luminosity exceeding $25 L^{*}$. These numbers may be compared with the value of $4 L^{*}$ found by Sandage (1973) to be the mean luminosity of brightest cluster members within his metric diameter. In general a large fraction of the central luminosity excess predicted by Fig. 1 may lie beyond Sandage's aperture.

The dynamical arguments reviewed above lead us to expect appreciable evolutionary effects on the galaxies near the centre of observed clusters. Although they do not give any direct predictions for the changes in metric magnitude of brightest cluster members, they do warn that substantial changes in the overall structure of such objects are inevitable. Until we achieve a better understanding of the dependence of the processes involved upon the properties both of the cluster and of the individual galaxies, there seems to be little chance of correcting the Hubble diagram for the effects of dynamical evolution. In the meantime a wide-ranging phenomenological or morphological study of galaxies in nearby clusters is more likely to lead to an understanding of evolutionary processes than is the measurement of the metric magnitudes of many distant objects. At present the future of the magnitude-redshift relation as a cosmological test looks bleak. Cosmological tests based on the apparent structure of 
the clusters themselves (e.g. those of Bahcall (1975) and of Fall (1976)) may have a better chance than tests based on the properties of individual cluster members, because the observed clustering may well be almost independent of both the stellar and the dynamical evolution of galaxies, whereas the dynamical evolution of an individual galaxy clearly depends strongly on the dynamics of the cluster in which it is embedded.

\section{REFERENCES}

Aarseth, S.J. and Lecar, M., 1975. Ann. Rev. Astron. Astrophys., 13, 1. Austin, T.B. and Peach, J.V., 1974. Mon. Not. R. astr. Soc., 167, 437. Bahcal1, N.A., 1975. Astrophys. J., 198, 249.

Chandrasekhar, S., 1942. Principles of SteZZar Dynomics, Dover, New York. Chandrasekhar, S., 1943. Astrophys. J., 97, 255.

Fal1, S.M., 1976. Mon. Not. R. astr. Soc., 176, 181.

Godwin, J. and Peach, J.V., 1976. Mon. Not. R. astr. Soc., in press.

Gunn, J.E. and Oke, J.B., 1975. Astrophys. J., 195, 255.

Gunn, J.E. and Tinsley, B.M., 1976. Astrophys. J., in press.

Kalnajs, A.J., 1970. in Gravitational N-body Problem (ed. Lecar, M.), Reide1, Dordrecht.

Lecar, M., 1974.-in Dynamias of StezZar Systems (ed. Hayli, A.), Reidel, Dordrecht.

Oemler, A., 1973. Astrophys. J., 180, 11.

Ostriker, J.P. and Tremaine, S.D., 1975. Astrophys. J., 202, L113.

Sandage, A., 1973. Astrophys. J., 183, 711.

Sandage, A., 1975. Astrophys. J., 202, 563.

Schechter, P., 1976. Astrophys. J., 203, 297.

Tremaine, S.D., Ostriker, J.P. and Spitzer, L., 1975. Astrophys. J., 196, 407.

Tremaine, S.D., 1976a, Astrophys. J., 203, 72.

Tremaine, S.D., 1976b, Astrophys. J., 203, 345.

White, S.D.M., 1976a, Mon. Not. R. astr. Soc., 174, 19.

White, S.D.M., 1976b, Mon. Not. R. astr. Soc., in press.

White, S.D.M., 1976c, Mon. Not. R. astr. Soc., in press. 
A. TOOMRE: You and Beatrice Tinsley have described very well what a confounded nuisance it is, for people who would like to know $q_{0}$, that galaxies eat galaxies. Yet in $\mathrm{N}$-body simulations you have yourself found that even clusters tend to eat clusters. Are you sure that this further complication will not eventually prove to be just as much of a nuisance for the various dynamical corrections - like for the comparisons with second and third-ranked galaxies that I think were recommended by Ostriker?

S. WHITE: I am quite sure that clusters do eat other clusters; however, I feel that we stand a much better chance of understanding the evolution of cannibalistic clusters than we do of understanding that of voracious galaxies in an evolving cluster environment. The physics which controls clustering is very simple, and $\mathrm{N}$-body experiments can give us a good idea of the way the lumpiness of the galaxy distribution and the characteristics of individual clusters may change with time. I think that at present it is hard to draw conclusions about evolution from a comparison of the Iuminosity functions of nearby and distant clusters as we do not have much idea of how a galaxy will look when it has finished digesting several of its companions.

S.M. FABER: A student at lick, A. Dossler, has measured luminosity functions for several rich clusters of galaxies. For four or five clusters with $\mathrm{CD}$ galaxies, he finds that the luminosity function is depressed at the bright end, as if the $\mathrm{CD}$ was formed at the expense of the other bright members. Although this result is in accordance with the dynamical friction model for the formation of cDs the total picture is not so encouraging. For example, there is at least one cluster with a depressed luminosity function but which contains no $\mathrm{cD}$, thus suggesting that, at least in some cases, such a peculiar luminosity function is primordial. Furthermore, amongst the other clusters without cDs, there is considerable variation in the luminosity function so that it would be extremely difficult to identify a universal primordial function which we could assume was present at the birth of the cluster. For this reason it is virtually impossible to test the dynamical friction mechanism quantitatively by estimating the changes to be expected in the luminosity function. Some sense may emerge from a much larger body of data. For the moment I am rather pessimistic about the amount which can be learned about dynamical corrections from a study of the upper end of the luminosity function in different clusters. 